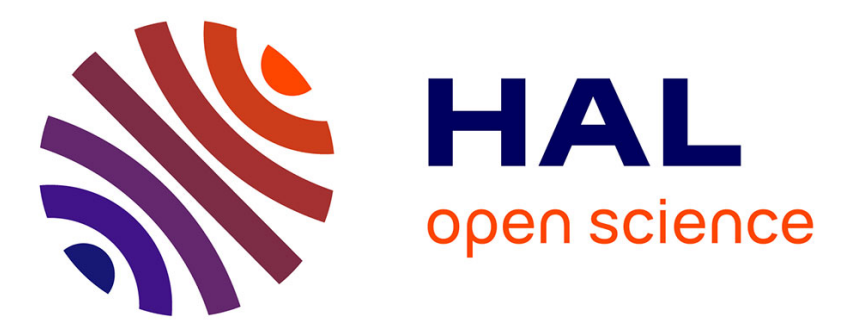

\title{
Estimation and correction of the modal damping error involving linear and nonlinear localized dissipation
}

Mohamed Krifa, Noureddine Bouhaddi, Gael Chevallier, Scott Cogan, Najib

Kacem

\section{- To cite this version:}

Mohamed Krifa, Noureddine Bouhaddi, Gael Chevallier, Scott Cogan, Najib Kacem. Estimation and correction of the modal damping error involving linear and nonlinear localized dissipation. European Journal of Mechanics - A/Solids, 2017, 66, pp.296 - 308. hal-02131305

\section{HAL Id: hal-02131305 \\ https://hal.science/hal-02131305}

Submitted on 16 May 2019

HAL is a multi-disciplinary open access archive for the deposit and dissemination of scientific research documents, whether they are published or not. The documents may come from teaching and research institutions in France or abroad, or from public or private research centers.
L'archive ouverte pluridisciplinaire HAL, est destinée au dépôt et à la diffusion de documents scientifiques de niveau recherche, publiés ou non, émanant des établissements d'enseignement et de recherche français ou étrangers, des laboratoires publics ou privés. 


\section{Estimation and correction of the modal damping error involving linear and nonlinear localized dissipation}

Article in European Journal of Mechanics - A/Solids · August 2017

DOI: 10.1016/j.euromechsol.2017.08.003

CITATIONS

0

5 authors, including:

\section{Mohamed Krifa}

University of Franche-Comté

8 PUBLICATIONS 3 CITATIONS

SEE PROFILE
READS

291

Some of the authors of this publication are also working on these related projects:

Noureddine Bouhaddi

University Bourgogne Franche-Comté

197 PUBLICATIONS 638 CITATIONS

SEE PROFILE 


\section{Accepted Manuscript}

Estimation and correction of the modal damping error involving linear and nonlinear localized dissipation

M. Krifa, N. Bouhaddi, G. Chevallier, S. Cogan, N. Kacem

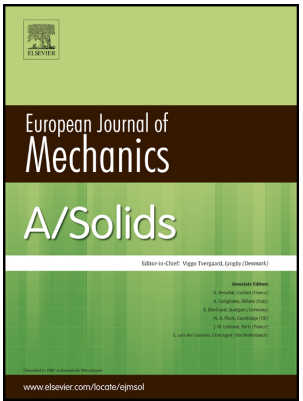

PII:

S0997-7538(16)30320-5

DOI:

10.1016/j.euromechsol.2017.08.003

Reference: EJMSOL 3471

To appear in: European Journal of Mechanics / A Solids

Received Date: 4 October 2016

Revised Date: 4 August 2017

Accepted Date: 6 August 2017

Please cite this article as: Krifa, M., Bouhaddi, N., Chevallier, G., Cogan, S., Kacem, N., Estimation and correction of the modal damping error involving linear and nonlinear localized dissipation, European Journal of Mechanics / A Solids (2017), doi: 10.1016/j.euromechsol.2017.08.003.

This is a PDF file of an unedited manuscript that has been accepted for publication. As a service to our customers we are providing this early version of the manuscript. The manuscript will undergo copyediting, typesetting, and review of the resulting proof before it is published in its final form. Please note that during the production process errors may be discovered which could affect the content, and all legal disclaimers that apply to the journal pertain. 


\title{
Estimation and Correction of the Modal Damping Error Involving Linear and Nonlinear Localized dissipation
}

\author{
M. Krifa, N. Bouhaddi ${ }^{1}$, G. Chevallier, S. Cogan, N. Kacem \\ Univ. Bourgogne Franche-Comte, FEMTO-ST Institute, CNRS/UFC/ENSMM/UTBM \\ Department of Applied Mechanics, 25000 BESANCON, FR
}

\begin{abstract}
The evaluation of damping in the assembled structures presents a real challenge. Indeed, because of the nonlinear behavior of the dissipation and their localization in interfaces, the equations of motion of a dynamic system involve coupling terms. These coupling terms are generally neglected, in engineering applications, by the community because of their complexity. Hence, a proportional damping is usually assumed even if it may provide inaccurate results. The aim of this paper is to investigate the assumptions related to modal damping in the cases of localized linear and nonlinear dissipation. To achieve this goal, a perturbation method based on an asymptotic expansion of the frequency response function is proposed. Two indices of nonlinear coupling and modal coupling are proposed in order to quantify and correct $a$ posteriori the error induced by modal damping assumption. Numerical examples are proposed in order to illustrate, first, the validation of the proposed method, by comparing the results with the reference solutions, and second, the usefulness of the proposed indices in quantifying and correcting errors induced by proportional damping hypothesis.
\end{abstract}

Keywords: Modal damping, Localized linear dissipation, Localized nonlinear dissipation, Modal coupling, Nonlinear coupling, Perturbation method

\footnotetext{
${ }^{1}$ corresponding author : noureddine.bouhaddi@univ-fcomte.fr
} 


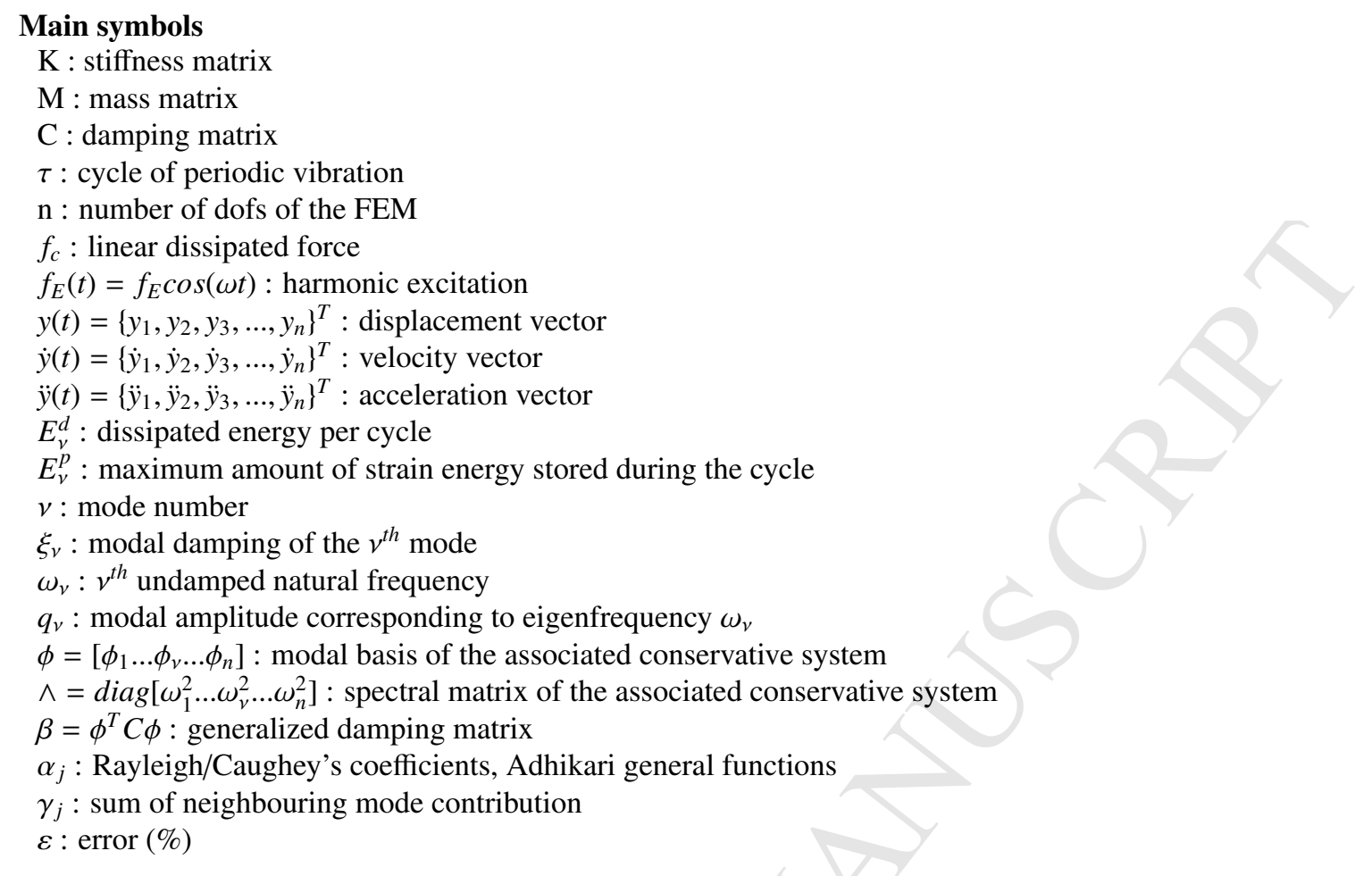

\section{Introduction}

Problems involving vibrations occur in many areas of mechanical, civil and aerospace engineering [1]. Vibrations are often undesirable and the interest lies in reducing them by introducing dampers. Since the publication of Lord Rayleigh [2], a large body of literature can now be found on damping. Although the topic of damping is an age old problem, the demands of modern engineering have led to a steady increase of interest in recent years $[3,4,5,6]$.

Damping presents one of the most important physical aspects to model and estimate, since it plays a large role in determining the amplitude of vibrations. Indeed, there are mainly two different kinds of damping in a structure [7] : (1) structural damping due to the internal dissipation in the material and (2) joint damping due to dissipation by friction between joint interfaces. The first kind of damping (structural damping) is widely known today for homogeneous structures but it is still the subject for research in composite structures. A number of experimental studies [8,9,10] have demonstrated that the damping resulting from interface effects is typically higher than the material damping in metallic structures. Consequently, the second kind of damping (joint damping) in the joint interface must be modeled in order to predict the vibration levels accurately. Damping in jointed interfaces is still not well mastered because of the complexity of the dissipation by friction which includes nonlinear behavior and localization effects [11]. Therefore, the eigenmodes will be coupled with damping. So the assumption of proportional damping can have limitations and may lead to significant errors in the case of most assembled structures.

Due to the dramatically different length scales associated with the dampers behavior and with the overall structure, the FRFs cannot be easily captured through direct numerical simulation including all physics, i.e. contact mechanics, material energy losses or fluid dynamics, and linear elastodynamics. For instance, bolted joints or welding points between parts induce physical phenomena similar to elasto-plasticity. Insulators induce viscous damping by energy losses in viscoelastic materials or by loss of pressure inside fluid components. Both technologies are responsible for localized, amplitude-dependent damping sources [12,13]. Because of its capacity of estimating the damping for both linear and nonlinear dissipation, Numerous works use the MSE method [1, 7, 14]. The advantage of MSE method is its capacity to compute modal damping by a real instead of a complex, eigenvalue solution. Consequently, the computational cost is greatly reduced. Recent works use an amplitude-dependent modal subspace to compute the damping ratio [13] or a Singular Value Decomposition of the modal subspace restricted to the joint neighborhood [15] to overcome the problems generated by the "proportional damping" assumption. The most practical method for 
accommodating the nonlinear nature of joint mechanisms within structural dynamic analysis is through metamodels of joints [3] employing natural dofs to the scale of structural dynamics, such as modal coordinates.

Numerous mathematical metamodels enabling the representation of such physical phenomena can be used in reduced order models for vibration study. Dhal's model [16] or Iwan's model [17] are available for friction joints. More recently, Segalman [18] proposed a four-parameter Iwan model that fits well with the experiments on jointed structures with only four parameters to identify. Friction induced damping can also be taken into account, less accurately but more easily, by using linear or nonlinear damping in the simulations [19].

Assemblies are not the only structures subjected to localized dissipation and nonlinearities. Moreover, most of the damping devices, polymer dampers, shunted piezoelectric, fluid dampers and electrostatic dampers are not uniformly distributed over the structures and behaves nonlinearly. For instance, in MicroElectroMechanical Systems (MEMS) and NanoElectroMechanical Systems (NEMS), the nonlinear damping effect cannot be easily separated from the linear one. Several experimental characterizations reported a significant increase in damping with respect to the actuation voltage in capacitive MEMS and NEMS [20,21]. Therefore, there is a need for consistent modeling of nonlinear electrodynamic damping which can be deduced from the quadratic Rayleigh dissipation function [22]. The resulting damping topology is of the Van Der Pol type for which the dissipation increases with respect to the vibration amplitude; this model is suitable for electrostatically coupled MEMS and NEMS [22, 23].

Although the development of many sophisticated dissipation models, damping is still not well mastered because of the neglect of coupling terms in the equations of motion of the discrete system. To the best of our knowledge, the impacts of nonlinear coupling and the modal coupling are still unknown and have yet to be identified in the literature.

The aim of this paper is to investigate the capabilities and limits of modal damping assumption in both cases of linear and nonlinear localized dissipation. In order to quantify and reduce the effects of the nonlinear and the modal coupling on the dynamic responses, an estimation and correction method based on perturbation approach [24, 25, 26] using assumption expansion of the responses is proposed. The limitations of the proportional damping assumption are then inspected and the correction of error estimations of modal damping due to the neglect of coupling terms is illustrated. Two indices of nonlinear coupling and modal coupling are proposed in order to quantify a posteriori the error induced by modal damping assumption. The proposed numerical examples are a 2-dof spring-mass system with localized nonlinear damper and a double-beam structure with a nonlinear interface, where accurate estimations of exact solutions are available.

\section{Limitation of the modal damping assumption}

The classical assumption of modal damping which consists of neglecting the coupling of eigenmodes through damping will be introduced and its validity range discussed. Next, the MSE method, which allows the estimation of the modal damping, will be presented briefly. Finally an illustrative example will be presented in both cases linear and nonlinear dissipation.

\subsection{Proportional damping assumption}

Damping is generally modeled under Rayleigh's [2] or Caughey's assumptions [27]. Rayleigh's assumption expresses damping as a linear combination of the mass and stiffness matrices,

$$
C=\alpha_{1} M+\alpha_{2} K
$$

The Rayleigh's (proportional) damping is a sufficient condition to obtain a diagonal generalized damping matrix ( $\beta=\phi^{T} C \phi$, where $\phi$ is the modal basis of the associated conservative system). Caughey introduced a necessary and sufficient condition

$$
C M^{-1} K=K M^{-1} C
$$

which leads to a diagonal generalized damping matrix. In the general case, the damping matrix is expressed as a power series of terms $\left(M^{-1} K\right)$ in the form

$$
C=M \sum_{j=0}^{N-1} \alpha_{j}\left(M^{-1} K\right)^{j}
$$


A further generalized form of proportional damping was proposed by Adhikari [28].

$$
C=M \alpha_{3}\left(M^{-1} K\right)=K \alpha_{4}\left(K^{-1} M\right)
$$

where $\alpha_{i}(\bullet), i=3, . .4$ are general functions.

These proportional damping assumptions are not always well-founded in reality, but leads to a diagonal generalized damping matrix that minimizes computational costs. The physical meaning of Caughey's and Rayleigh's assumptions is that dissipation in the structure is uniformly distributed so that modes are decoupled with respect to damping effects. But in reality, the big amount of damping is localized in interfaces of assembled structures. The present study will investigate the limits of the proportional assumption for structures with non-proportional linear and nonlinear dissipative interfaces.

\subsection{Modal Strain Energy method (MSE)}

In order to estimate the modal damping of a structure, the Modal Strain Energy method (MSE) can be used. It was firstly suggested by Ungar and Kerwin [29], and has been used since to address viscoelastic damping problems of sandwich structures by Johnson and Klenholz [30]. Later, a modified MSE was proposed [31] in order to improve the estimation of modal damping. The objective of the modal strain energy is to determine the damping factor corresponding to each vibration mode of the structure. It is based on the concept of the dissipated energy in the interfaces for which the close form expression of the loss factor is the ratio between dissipated energy $E_{v}^{d}$ and maximal potential energy $E_{v}^{p}$, over a cycle of periodic vibration [32], as shown in this relation:

$$
\xi_{v}=\frac{1}{4 \pi} \frac{E_{v}^{d}}{E_{v}^{p}}
$$

\subsection{Illustrative example}

The aim of this section is to illustrate the limitation of the modal damping assumption. This limitation is due to the importance of the coupling linear and nonlinear damping terms. Figure 1 shows a first model of a two-dof system with localized nonlinear dissipation force between the two dofs. In order to model the nonlinear dissipation in the interface a localized Van der Pol force is considered. And, in order to model the internal linear dissipation a viscous force model is considered. So, in the following, a non-proportional linear and nonlinear damping will be investigated.

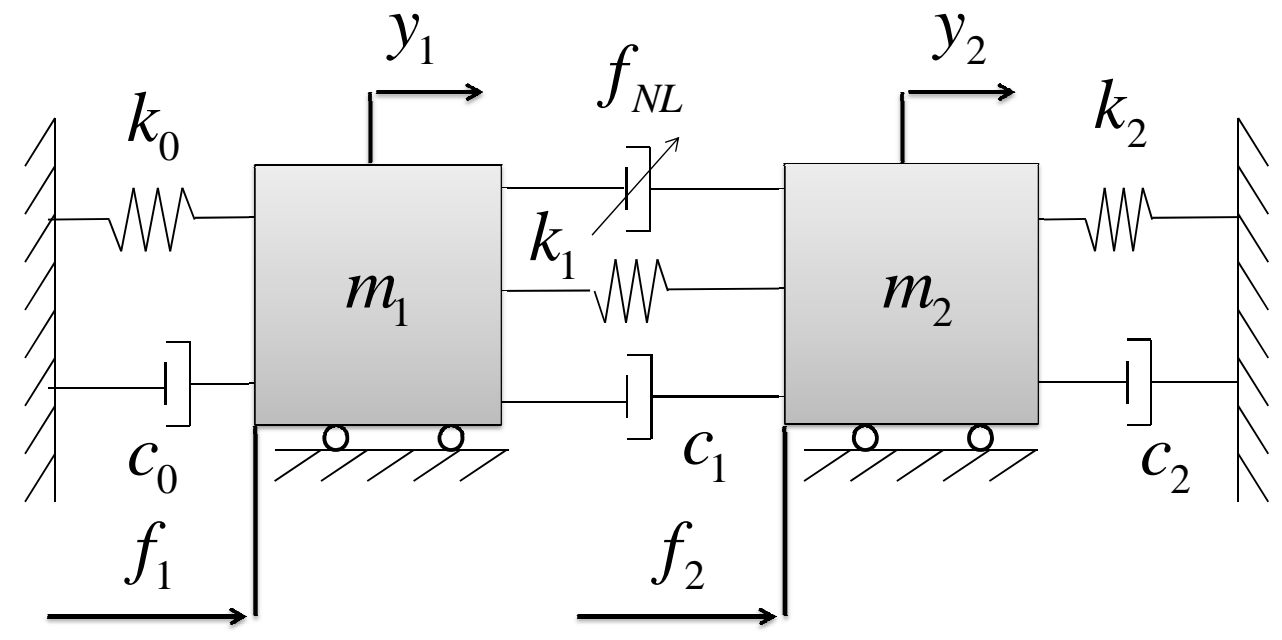

Figure 1: Non-proportional nonlinear 2 DOFs system

A harmonic excitation force $f_{E}=\left\{\begin{array}{ll}f_{1} & f_{2}\end{array}\right\}^{T}$ is applied to nodes of the 2 DOFs system, where the values of the excitation amplitudes are known.

The formulations of the parameter matrices are given as : 


$$
M=\left[\begin{array}{cc}
m_{1} & 0 \\
0 & m_{2}
\end{array}\right], K=\left[\begin{array}{cc}
k_{0}+k_{1} & -k_{1} \\
-k_{1} & k_{1}+k_{2}
\end{array}\right], C=\left[\begin{array}{cc}
c_{0}+c_{1} & -c_{1} \\
-c_{1} & c_{1}+c_{2}
\end{array}\right]
$$

The linear and nonlinear dissipated forces are given as :

$$
f_{D}=f_{\mathrm{LN}}+f_{\mathrm{NL}}
$$

where

$$
f_{\mathrm{LN}}=C \dot{y}=\left[\begin{array}{cc}
c_{0}+c_{1} & -c_{1} \\
-c_{1} & c_{1}+c_{2}
\end{array}\right]\left\{\begin{array}{l}
\dot{y}_{1} \\
\dot{y}_{2}
\end{array}\right\} \text { and } f_{\mathrm{NL}}=\left[\begin{array}{c}
\alpha y_{\text {rel }}{ }^{2} \dot{y}_{\text {rel }} \\
-\alpha y_{\text {rel }}{ }^{2} \dot{y}_{\text {rel }}
\end{array}\right]
$$

where $y_{r e l}=y_{1}-y_{2}$ is the relative response of the system, $\dot{y}_{r e l}=\dot{y}_{1}-\dot{y}_{2}$ the corresponding relative velocity.

The values of the physical elements of the linear damped system are : $m_{1}=m_{2}=1 \mathrm{Kg}, c_{0}=0.05 \mathrm{Ns} / \mathrm{m} ; c_{1}=0$ $\mathrm{Ns} / \mathrm{m} ; c_{2}=0.3 \mathrm{Ns} / \mathrm{m}, k_{0}=4 \mathrm{~N} / \mathrm{m} ; k_{1}=1 \mathrm{~N} / \mathrm{m} ; k_{2}=4 \mathrm{~N} / \mathrm{m} ;\left|f_{1}\right|=1 N ;\left|f_{2}\right|=0 N$.

These parameters have been chosen in order to have closed modes and localized dissipation. Table 1 summarizes the corresponding natural frequencies and damping ratios, of the 2-dof system, which are calculated by the state space method.

Table 1: Natural frequencies and damping ratios of a 2-dof system

\begin{tabular}{ccc}
\hline Mode & 1 & 2 \\
\hline Frequency $(\mathrm{Hz})$ & 0.32 & 0.39 \\
Damping $\xi(\%)$ & 4.39 & 3.56 \\
\hline
\end{tabular}

Equation (9) gives the corresponding generalized damping matrix $\beta$. The presence of extra-diagonal terms causes the modal coupling.

$$
\beta=\left(\begin{array}{cc}
0.175 & -0.125 \\
-0.125 & 0.175
\end{array}\right)
$$

\subsection{Discussion}

Two test cases will be illustrated in this part: (a) case of non-proportional linear damping when $f_{N L}=0$, (b) case of non-proportional nonlinear damping when $f_{N L} \neq 0$.

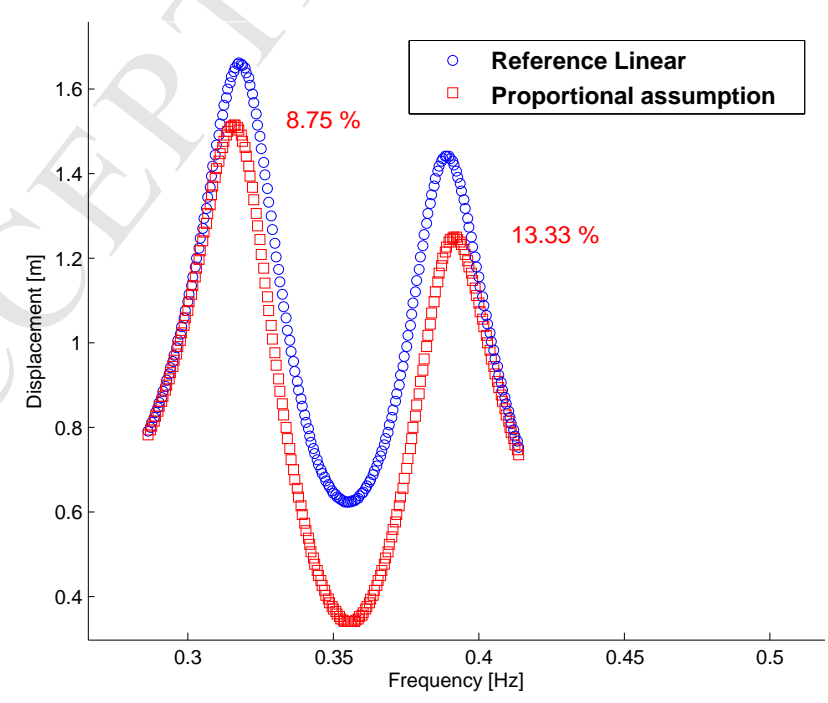

Figure 2: Frequency response of the linear model: $\circ$ Reference, $\square$ Proportional damping 
(a) In the case of non-proportional linear dissipation, the two modes of the system are coupled due to the presence of extra-diagonal terms in the generalized damping matrix. The Figure 2 presents the frequency response of the 2 dof system. The blue curve is the reference response, it is calculated using the state space method. The red curve is the estimated response using the proportional damping assumption. It can be seen that the case of proportional damping, which don't take into account couplings, appears to be insufficient and presents amplitude errors and phase shift in the case of non-proportional linear damping. If the coupling terms are neglected, the level response shows an error of $8.75 \%$ for the first mode and $13.33 \%$ for the second mode. Now what will happen, if the nonlinear behavior of the dissipated force is introduced to the model ?

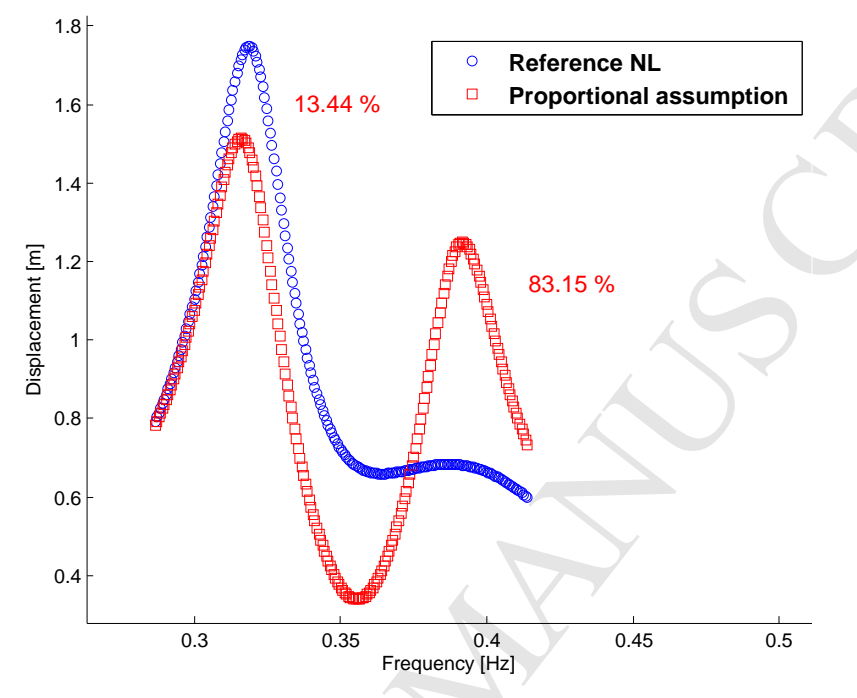

Figure 3: Frequency response of the nonlinear model : ० Reference, $\square$ Proportional damping

(b) In the case of non-proportional nonlinear dissipation, eigenmodes are coupled due to both modal and nonlinear coupling terms of the damping. The Figure 3 presents the comparison of frequency responses between the nonlinear reference solution and the proportional damping estimation. The blue curve is the nonlinear reference response, it is calculated using a time integration method and the Fast Fourier transform. The red curve is the estimated response using the proportional damping assumption. From Figure 3 it can be seen that the maximum level error increases and may reach the values, $13.44 \%$ for the first mode and $83.15 \%$ for the second mode, when neglecting the nonlinear coupling terms.

The important question to address here is just how can we predict and reduce these level errors ? In the following, a perturbation method will be proposed in order to get more accurate results when taking into account both the modal and nonlinear coupling terms. 


\section{Estimation and correction method involving nonlinear localized dissipation}

\subsection{Proposed method}

We propose an extension of perturbation technique $[24,25,26]$ for a multi-degree of freedom (MDOF). The scope of the proposed method is that it takes into account the effects of coupling including linear and nonlinear terms. The perturbation method or the asymptotic method is applicable to problems in which a small positive non-dimensional bookkeeping parameter $\varepsilon$ is associated with the coupling term of the differential equation. If the solution of the linearized problem is periodic, and if $\varepsilon$ is small, we can expect the perturbed solution to be periodic also. The perturbation method will be explained more fully here after. The equations governing the dynamic response of the MDOF system can be expressed in the following form,

$$
M \ddot{y}+f_{D}(y, \dot{y})+K y=f_{E}(t)
$$

where $f_{E}(t)=F_{E} \cos (\Omega t)$ is a harmonic excitation with an excitation frequency $\Omega, M$ and $K$ are respectively the mass and the stiffness matrix of the system. $f_{D}(y, \dot{y})$ is the dissipated force vector of the system, which includes the material dissipation and principally the dissipation due to the interfaces between the substructures.

Let's consider $\phi$ and $\Lambda$ respectively the modal base and the spectral matrix of the eigensolution associated to the conservative system $K \phi=M \phi \Lambda$, where : $\phi^{T} M \phi=I, \phi^{T} K \phi=\Lambda$.

By projection on the modal base $\Phi$ truncated at the first $m$ modes and multiplying on the left by $\Phi^{T}$, Equation (10) in modal coordinates $q$ yields

$$
\ddot{q}+F_{D}(q, \dot{q})+\Lambda q=F_{E}(t)
$$

where $F_{D}(q, \dot{q})=\phi^{T} f_{D}(q, \dot{q})$ is the modal nonlinear forces due to friction contacts and $F_{E}(t)=\phi^{T} f_{E}(t)$ is the generalized excitation forces. The above system presents $m$ coupled nonlinear differential equations because of the presence of the dissipated force $F_{D}(q, \dot{q})$. In order to investigate both modal coupling effect and the nonlinear effect, a double perturbation method is considered.

This method consists in decomposing the dissipation force into two parts according to the type of dissipation : linear and nonlinear. Each part is also decomposed into two parts : uncoupled and coupled. So, the dissipation force can be expressed as follows :

$$
\phi^{T} f_{D}(q, \dot{q})=\underbrace{\phi_{\text {Uncoupled }}^{\phi^{T} f_{D}^{U L}(q, \dot{q})}+\varepsilon_{2} \underbrace{\phi^{T} f_{D}^{C L}(q, \dot{q})}_{\text {Coupled }}}_{\text {Linear }}+\varepsilon_{1}^{(\varepsilon_{\text {Uncoupled }}^{\phi^{T} f_{D}^{U N L}(q, \dot{q})}+\varepsilon_{2} \underbrace{\varepsilon^{T} f_{D}^{C N L}(q, \dot{q})}_{\text {Nonlinear }})}
$$

where $\phi^{T} f_{D}{ }^{U L}, \phi^{T} f_{D}{ }^{C L}$ are respectively the uncoupled and coupled linear dissipation force, and $\phi^{T} f_{D}{ }^{U N L}, \phi^{T} f_{D}{ }^{C N L}$ are respectively the uncoupled and coupled nonlinear dissipation force. $\varepsilon_{1}$ and $\varepsilon_{2}$ are two small positive nondimensional bookkeeping parameters.

From Equation (12) one can distinguish three particular cases :

(a) when $\varepsilon_{1}=0$, the parameter $\varepsilon_{2} \ll 1$ allows to study the effects of the linear modal coupling.

(b) when $\varepsilon_{2}=0$, the parameter $\varepsilon_{1} \ll 1$ allows to study the effects of the uncoupled nonlinearity.

(c) when both parameters $\varepsilon_{1} \neq 0$ and $\varepsilon_{2} \neq 0$, both effects of modal coupling and nonlinearity can be investigated.

Substituting Equation (12) into (11) yields

$$
\ddot{q}+\Lambda q+\phi^{T} f_{D}^{U L}(q, \dot{q})+\varepsilon_{2} \phi^{T} f_{D}^{C L}(q, \dot{q})+\varepsilon_{1}\left(\phi^{T} f_{D}^{U N L}(q, \dot{q})+\varepsilon_{2} \phi^{T} f_{D}^{C N L}(q, \dot{q})\right)=\phi^{T} f_{E}(t)
$$

For the $k^{\text {th }}$ mode, Equation (13) yields

$\ddot{q}_{k}+\omega_{k}^{2} q_{k}+\phi^{T} f_{D_{k}}^{U L}\left(q_{k}, \dot{q}_{k}\right)+\varepsilon_{2} \phi^{T} f_{D_{k}}^{C L}\left(q_{l \neq k}, \dot{q}_{l \neq k}\right)+\varepsilon_{1}\left(\phi^{T} f_{D_{k}}^{U N L}\left(q_{k}, \dot{q}_{k}\right)+\varepsilon_{2} \phi^{T} f_{D_{k}}^{C N L}\left(q_{l \neq k}, \dot{q}_{l \neq k}\right)\right)=\phi^{T} f_{E_{k}}(t)$

For $\varepsilon_{1} \ll 1$ and $\varepsilon_{2} \ll 1$, one assumes that the perturbation solution of Equation (14) is sought in the form 


$$
q=\sum_{i, j} \varepsilon_{1}^{i} \varepsilon_{2}^{j} q^{(i, j)}=q^{(0,0)}+\varepsilon_{1} q^{(1,0)}+\varepsilon_{1} \varepsilon_{2} q^{(1,1)}+\varepsilon_{2} q^{(0,1)}+\cdots
$$

where $q^{(0,0)}$ is a Linear Uncoupled response that corresponds to the proportional assumption, $q^{(0,1)}$ is a Linear Coupled response, $q^{(1,0)}$ is a NonLinear Uncoupled response and $q^{(1,1)}$ is a NonLinear Coupled response.

Substituting Equation (15) into (14) yields

$$
\begin{aligned}
& \left(\ddot{q}_{k}^{(0,0)}+\varepsilon_{1} \ddot{q}_{k}^{(1,0)}+\varepsilon_{1} \varepsilon_{2} \ddot{q}_{k}^{(1,1)}+\varepsilon_{2} \ddot{q}_{k}^{(0,1)}+\cdots\right)+\omega_{k}^{2}\left(q_{k}^{(0,0)}+\varepsilon_{1} q_{k}^{(1,0)}+\varepsilon_{1} \varepsilon_{2} q_{k}^{(1,1)}+\varepsilon_{2} q_{k}^{(0,1)}+\cdots\right) \\
& +\phi^{T} f_{D_{k}}^{U L}\left(q_{k}^{(0,0)}+\varepsilon_{1} q_{k}^{(1,0)}+\cdots, \dot{q}_{k}^{(0,0)}+\varepsilon_{1} \dot{q}_{k}^{(1,0)}+\cdots\right)+\varepsilon_{2} \phi^{T} f_{D_{l \neq k}}^{C L}\left(q_{l \neq k}^{(0,0)}+\varepsilon_{1} q_{l \neq k}^{(1,0)}+\cdots, \dot{q}_{l \neq k}^{(0,0)}+\varepsilon_{1} \dot{q}_{l \neq k}^{(1,0)}+\cdots\right) \\
& +\varepsilon_{1}\left(\phi^{T} f_{D_{k}}^{U N L}\left(q_{k}^{(0,0)}+\varepsilon_{1} q_{k}^{(1,0)}+\cdots, \dot{q}_{k}^{(0,0)}+\varepsilon_{1} \dot{q}_{k}^{(1,0)}+\cdots\right)+\varepsilon_{2} \phi^{T} f_{D_{l \neq k}^{C N L}}^{C N L}\left(q_{l \neq k}^{(0,0)}+\varepsilon_{1} q_{l \neq k}^{(1,0)}+\cdots, \dot{q}_{l \neq k}^{(0,0)}+\varepsilon_{1} \dot{q}_{l \neq k}^{(1,0)}+\cdots\right)\right)=\phi^{T} f_{E_{k}}(t)
\end{aligned}
$$

Regrouping terms of the same order gives

Order $(\mathbf{0 , 0}), \varepsilon_{1}=0$ and $\varepsilon_{2}=0$ : Proportional assumption

$$
\ddot{q}_{k}^{(0,0)}+\omega_{k}^{2} q_{k}^{(0,0)}+\phi^{T} f_{D_{k}}^{U L}\left(q_{k}^{(0,0)}, \dot{q}_{k}^{(0,0)}\right)=\phi^{T} f_{E_{k}}(t)
$$

Order $(\mathbf{0 , 1}), \varepsilon_{1}=0$ and $\varepsilon_{2} \neq 0$ : Effect of modal coupling

$$
\ddot{q}_{k}^{(0,1)}+\omega_{k}{ }^{2} q_{k}{ }^{(0,1)}+\phi^{T} f_{D_{k}}^{U L}\left(q_{k}{ }^{(0,1)}, \dot{q}_{k}^{(0,1)}\right)=-\phi^{T} f_{D_{k}}^{C L}\left(q_{l \neq k}{ }^{(0,0)}, \dot{q}_{l \neq k}^{(0,0)}\right)
$$

Order $\left(\mathbf{1 , 0 )}, \varepsilon_{1} \neq 0\right.$ and $\varepsilon_{2}=0$ : Effect of uncoupled nonlinearity

$$
\ddot{q}_{k}^{(1,0)}+\omega_{k}^{2} q_{k}^{(1,0)}+\phi^{T} f_{D_{k}}^{U L}\left(q_{k}^{(1,0)}, \dot{q}_{k}^{(1,0)}\right)=-\phi^{T} f_{D_{k}}^{U N L}\left(q_{k}^{(0,0)}, \dot{q}_{k}^{(0,0)}\right)
$$

Order $(\mathbf{1 , 1}), \varepsilon_{1} \neq 0$ and $\varepsilon_{2} \neq 0$ : Effect of coupled nonlinearity

$$
\begin{aligned}
& \ddot{q}_{k}^{(1,1)}+\omega_{k}^{2} q_{k}^{(1,1)}+\phi^{T} f_{D_{k}}^{U L}\left(q_{k}^{(1,1)}, \dot{q}_{k}^{(1,1)}\right)=-\phi^{T} f_{D_{k}}^{C L}\left(q_{l \neq k}^{(1,0)}, \dot{q}_{l \neq k}^{(1,0)}\right) \\
& -\phi^{T} f_{D_{k}}^{U N L}\left(q_{k}^{(0,1)}, \dot{q}_{k}^{(0,1)}\right)-\phi^{T} f_{D_{k}}^{C N L}\left(q_{l \neq k}^{(0,0)}, \dot{q}_{l \neq k}^{(0,0)}\right)
\end{aligned}
$$

From the order $(1,1)$, the double perturbation method takes into account both the nonlinearity and modal coupling effects. The main advantage of this method of double perturbation consists in solving linear uncoupled equations at each order. The nonlinear terms are taken into account in the right hand side of the resulting equation at each order and depends only on the solutions at the previous orders.

\subsection{Particular case of linear damping}

The aim of this section is to present the formulation of the perturbation method in the particular case of linear damping.

It is a common practice to approximate the nonlinear behavior with an equivalent linear damping and not conduct a nonlinear analysis [33]. Because of its simplicity, the equivalent viscous model will be considered here [34]. From Equation (10), one can deduces the discrete form of the damped linear vibration problem which is governed by the following equation

$$
M \ddot{y}+C \dot{y}+K y=f_{E}(t)
$$

where $f_{D}(y, \dot{y})=C \dot{y}$, and $C$ is the viscous damping matrix.

Assuming that the viscous damping matrix $\mathrm{C}$ is a non-proportional matrix, which represent the case of most assembled structures where the dissipation is principally distributed in the interfaces, then the generalized damping matrix $\beta=\phi^{T} C \phi$ will be a full matrix. In the particular case of linear dissipation, for simplification reasons, the following notations will be considered : $\varepsilon_{1}=0$ and $\varepsilon_{2}=\varepsilon$, the order $(0,0)=(0)$, the order $(0,1)=(1)$ and the order $(0, n)=(n)$, ect...

Given $\varepsilon \in[0,1]$, the perturbation method consists in expressing the generalized damping matrix $\beta$ in the form:

$$
\beta=\beta_{1}+\varepsilon \beta_{2}
$$

where $\beta_{1}$ is the diagonal part of the matrix $\beta$ and $\beta_{2}$ contains the off-diagonal terms with zeros on the diagonal. 
If $\varepsilon=0$, then Equation (22) gives $\beta=\beta_{1}$, which corresponds to the case of proportional damping. Otherwise, if $\varepsilon=1$ then Equation (22) gives $\beta=\beta_{1}+\beta_{2}$, corresponding to the general case of localized damping without added assumptions.

For $\varepsilon \ll 1$, one assumes that the perturbation solution of the Equation (21) is sought in the form

$$
y_{n}(t)=y^{(0)}+\varepsilon y^{(1)}+\varepsilon^{2} y^{(2)}+\cdots+\varepsilon^{n} y^{(n)}
$$

Assume that the perturbation terms $y^{(i)}$ of the $i^{\text {th }}$ order projected on the modal basis are expressed as follow :

$$
y^{(i)}=\phi q^{(i)}
$$

Each perturbation term $y^{(i)}$, which is complex, is expressed as the multiplication of the modal basis $\phi$, which is real, and the modal coordinate $q^{(i)}$ which is complex.

Substituting Equation (24) into (23) yields

$$
y_{n}(t)=\phi\left(q^{(0)}+\varepsilon q^{(1)}+\varepsilon^{2} q^{(2)}+\ldots+\varepsilon^{n} q^{(n)}\right)
$$

Now, the perturbation terms $q^{(i)}$ of the $i^{\text {th }}$ order need to be derived.

Substituting Equation (25) into Equation (21) and multiplying the two sides of the equality by $\phi^{T}$ on the left yields:

$$
\phi^{T} M \phi\left(\ddot{q}^{(0)}+\varepsilon \ddot{q}^{(1)}+\varepsilon^{2} \ddot{q}^{(2)}+\cdots\right)+\phi^{T} C \phi\left(\dot{q}^{(0)}+\varepsilon \dot{q}^{(1)}+\varepsilon^{2} \dot{q}^{(2)}+\cdots\right)+\phi^{T} K \phi\left(q^{(0)}+\varepsilon q^{(1)}+\varepsilon^{2} q^{(2)}+\cdots\right)=\phi^{T} f_{E}
$$

The orthonormality conditions $\phi^{T} M \phi=I, \phi^{T} K \phi=\Lambda, \phi^{T} C \phi=\beta$ lead to the following expression:

$$
\ddot{q}^{(0)}+\varepsilon \ddot{q}^{(1)}+\varepsilon^{2} \ddot{q}^{(2)}+\cdots+\left(\beta_{1}+\varepsilon \beta_{2}\right)\left(\dot{q}^{(0)}+\varepsilon \dot{q}^{(1)}+\varepsilon^{2} \dot{q}^{(2)}+\cdots\right)+\Lambda q^{(0)}+\varepsilon \Lambda q^{(1)}+\varepsilon^{2} \Lambda q^{(2)}+\cdots=\phi^{T} f_{E}
$$

Regrouping terms of the same order gives

$$
\ddot{q}^{(0)}+\beta_{1} \dot{q}^{(0)}+\Lambda q^{(0)}+\varepsilon\left(\ddot{q}^{(1)}+\beta_{1} \dot{q}^{(1)}+\Lambda q^{(1)}+\beta_{2} \dot{q}^{(0)}\right)+\varepsilon^{2}\left(\ddot{q}^{(2)}+\beta_{1} \dot{q}^{(2)}+\Lambda q^{(2)}+\beta_{2} \dot{q}^{(1)}\right)+\varepsilon^{3} \beta_{2} \dot{q}^{(2)}+\cdots=\phi^{T} f_{E}
$$

From the order 0 in $\varepsilon$ the expression $\ddot{q}^{(0)}+\beta_{1} \dot{q}^{(0)}+\Lambda q^{(0)}=\phi^{T} f_{E}$ can be derived and the perturbation term $q^{(0)}$ is calculated as follows:

$$
q^{(0)}=\left(-\omega^{2} I+j \omega \beta_{1}+\Lambda\right)^{-1} \phi^{T} f_{E}
$$

The response $q^{(0)}$ corresponds to the initial unperturbed system. This response is calculated once in the beginning of the procedure and the remaining higher order terms $q^{(i)}$ are expressed as a function of $q^{(0)}$.

The $1^{\text {st }}$ order terms in $\varepsilon$ of Equation (28) lead to the expression $\ddot{q}^{(1)}+\beta_{1} \dot{q}^{(1)}+\Lambda q^{(1)}=-\beta_{2} \dot{q}^{(0)}$, so the perturbation term $q^{(1)}$ as a function of $q^{(0)}$ is calculated as follows:

$$
q^{(1)}=-j \omega\left(-\omega^{2} I+j \omega \beta_{1}+\Lambda\right)^{-1} \beta_{2} q^{(0)}
$$

Similarly, the $2^{\text {nd }}$ order terms in $\varepsilon$ lead to $\ddot{q}^{(2)}+\beta_{1} \dot{q}^{(2)}+\Lambda q^{(2)}=-\beta_{2} \dot{q}^{(1)}$ and the perturbation term $q^{(2)}$ expressed as a function of $q^{(1)}$ is given by:

$$
q^{(2)}=-j \omega\left(-\omega^{2} I+j \omega \beta_{1}+\Lambda\right)^{-1} \beta_{2} q^{(1)}
$$

In general, the $n^{\text {th }}$ order term in $\varepsilon$ allows $q^{(n)}$ to be expressed as a function of $q^{(n-1)}$ or even $q^{(0)}$ as follows:

$$
q^{(n)}=-j \omega\left(-\omega^{2} I+j \omega \beta_{1}+\Lambda\right)^{-1} \beta_{2} q^{(n-1)}=\psi q^{(n-1)}=\psi^{n} q^{(0)}
$$

where $\psi=-j \omega\left(-\omega^{2} I+j \omega \beta_{1}+\Lambda\right)^{-1} \beta_{2}$ is a matrix depending on $\beta_{1}$ and $\beta_{2}$.

The $0^{\text {th }}$ order term with $(\varepsilon=0)$ yields the unperturbed FRF resulting from a diagonal generalized damping and is given by the classical expression:

$$
y_{0}(\omega)=\phi q_{0}=\phi\left(-\omega^{2} I+j \omega \beta_{1}+\Lambda\right)^{-1} \phi^{T} f_{E}
$$


Moreover, the perturbed response of order $n$ can be expressed as:

$$
y_{n}(\omega)=\phi\left(1+\varepsilon \psi+\varepsilon^{2} \psi^{2}+\cdots+\varepsilon^{n} \psi^{n}\right) q_{0}
$$

It should be noted that the matrix $\psi$ is calculated once for each frequency $\omega$. The main advantage of the expression (34) is its capacity to calculate, for a very low computational cost, any order of the perturbation to improve accuracy. The low cost is explained, first by the inversion of diagonal matrix $\beta_{1}$ in Equation (32). Second, whatever the size of the model governed by Equation (21), the matrix is equal in size to the number of retained modes. To validate the perturbation method, the results will be compared to the direct reference method using full matrices:

$$
y(\omega)=\phi\left(-\omega^{2} I+j \omega\left(\beta_{1}+\varepsilon \beta_{2}\right)+\Lambda\right)^{-1} \phi^{T} f_{E}
$$

The proposed perturbation method will be illustrated with two numerical examples in order to investigate the importance of the off-diagonal terms in the generalized damping matrix on the frequency response of the structure.

\subsubsection{Quantification indices}

In order to quantify the extent of non-proportionality, several indices have been proposed in the literature for quantification of the damping non-proportionality [35]. Bhaskar [36] has proposed a non-proportionality index based on the error introduced by ignoring the coupling terms in the modal damping matrix. He has analyzed the behavior of errors in FRF when the off-diagonal terms of modal damping matrix are neglected. An analytical index for the quantification of non-proportionality for discrete vibrating systems was developed in [37]. Finally, it should be noted that FRF of viscously damped systems with non-proportional damping can be obtained exactly in terms of the complex frequencies and complex modes using state space method [38]. In this paper two indices are proposed and taken into account :

(1) nonlinear coupling index

(2) modal coupling index

$$
I_{N L}=100 \times \frac{\left\|F_{N L}\right\|_{\infty}}{\left\|F_{D}\right\|_{\infty}}
$$

$$
I_{L N}=100 \times \frac{\left\|F_{C}\right\|_{\infty}}{\left\|F_{L N}\right\|_{\infty}}
$$

The first ratio indicates the contribution of the nonlinear force $F_{N L}$ in the total dissipation force $F_{D}=F_{L N}+F_{N L}$. The second ratio indicates the contribution of the coupled force $F_{C}$ in the linear dissipation force $F_{L N}=F_{C}+F_{U}$.

The effects of both the nonlinear coupling and modal coupling on the level responses will be investigated.

The amplitude errors between two responses $y_{1}$ and $y_{2}$, in the frequency band of interest, are given by:

$$
\varepsilon_{y}=100 \times \frac{\left\|y_{2}-y_{1}\right\|}{\left\|y_{1}\right\|}
$$

In the linear case, a residual error for the approximate solution can be evaluated a posteriori without calculating the reference solution. By injecting the response obtained in Equation (34) into the full problem described by Equation (21) one can get: $\left(K+j \omega C-\omega^{2} M\right) \times y_{n}(\omega)=f_{E}+\Delta f_{E}$ where $\Delta f_{E}$ is the residual force that should be minimised. A convergence indicator $\varepsilon_{f}$ is proposed based on the ratio between the residual energy $y_{n}^{T} \Delta f_{E}$ and the potential energy $y_{n}^{T} K y_{n}$ as follows:

$$
\varepsilon_{f}=\frac{y_{n}^{T} \Delta f_{E}}{y_{n}^{T} K y_{n}}
$$

Thus, no knowledge of the exact solution is required. The order of perturbation is increased in order to obtain $\varepsilon_{f}<\varepsilon_{\text {limit }}$, where $\varepsilon_{\text {limit }}$ is a given accuracy fixed a priori. 


\section{Numerical simulations}

\subsection{Application to a 2-DOF system}

A first mechanical system, shown in section 2.3, consisting of 2-dofs model, is used to illustrate the effectiveness of the proposed perturbation method. As shown in Figure 4, the order $(0,0)$ which corresponds to the proportional damping hypothesis has a response level error equals to $13.44 \%$. This error decreases if we consider the linear coupling. The order (0.1) presents an error equals to $5.55 \%$. Taking into account the presence of uncoupled nonlinearity, the response is clearly corrected and the error of the order (1.0) becomes 5.03\%. Finally, if the coupled nonlinearity is considered, the order $(1,1)$ presents in this case only $3.32 \%$ of error with respect to the nonlinear reference.

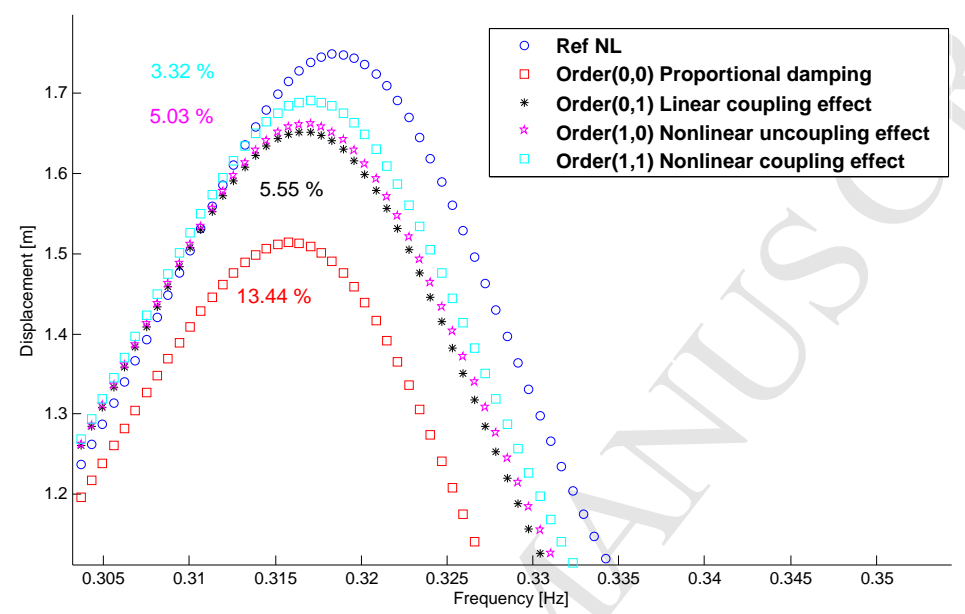

Figure 4: Frequency response of the nonlinear model, nonlinear coupling index $I_{N L}=20 \%$, focus on the first mode: $\circ$ Reference, $\square$ Proportional damping, * Order $(0,1)$ - Linear coupling effect, $\star$ Order $(1,0)$ - nonlinear uncoupling effect, $\square$ Order $(1,1)$ - nonlinear coupling effect

A parametric study was carried out subsequently in order to investigate the impact of the nonlinearity. The ratio $I_{N L}$ is able to indicate the percentage of the nonlinear force relative to the total dissipation force. Six levels of the nonlinear force relative to the overall dissipation of strength were studied. The corresponding error is calculated for different perturbation orders. From Table 2 it can be seen that, for a given order $((0,0),(0,1)$ or $(1,0))$ the error increases with the increase of the index of the nonlinearity dissipation $I_{N L}$. However, for the order $(1,1)$ when considering the modal and nonlinear coupling, the error remains less than $3 \%$ for low nonlinearity $\left(I_{N L} \leq 20 \%\right)$.

Therefore, the proposed method based on a double perturbation formulation is able to correct both amplitude and phase shift when switching to higher orders. The order $(1,1)$ is sufficient and can generate responses with good accuracy (less than $4 \%$ of level error) for high nonlinearity $\left(I_{N L}=30 \%\right)$.

Table 2: Amplitude Error (\%)

\begin{tabular}{cccc||c||cc}
\hline Ratio $I_{N L}$ & 5 & 10 & 15 & 20 & 25 & 30 \\
\hline$(0,0)$ & $\mathbf{9 . 8 8}$ & $\mathbf{1 0 . 9 4}$ & $\mathbf{1 1 . 8 8}$ & $\mathbf{1 3 . 4 4}$ & $\mathbf{1 4 . 6 0}$ & $\mathbf{1 5 . 1 3}$ \\
$(0,1)$ & 1.66 & 2.82 & 3.78 & 5.55 & 6.82 & 7.40 \\
$(1,0)$ & 1.54 & 2.58 & 3.49 & 5.03 & 6.09 & 6.55 \\
$(1,1)$ & $\mathbf{1 . 2 5}$ & $\mathbf{1 . 9 4}$ & $\mathbf{2 . 4 4}$ & $\mathbf{3 . 3 2}$ & $\mathbf{3 . 7 8}$ & $\mathbf{3 . 9 2}$ \\
\hline
\end{tabular}




\subsection{Application to a double-beam system}

After validating the proposed method on a first model of two dofs system, a second model will be studied in the following. As shown in Figure 5, the considered structure is composed of two beams. Each beam is discretized into ten $2 \mathrm{D}$ beam finite elements ( 2 dofs per node), so the full model has 40 dofs. The excitation force is applied to node number 1 of the first beam. This model represents two steel substructures assembled by a nonlinear joint.

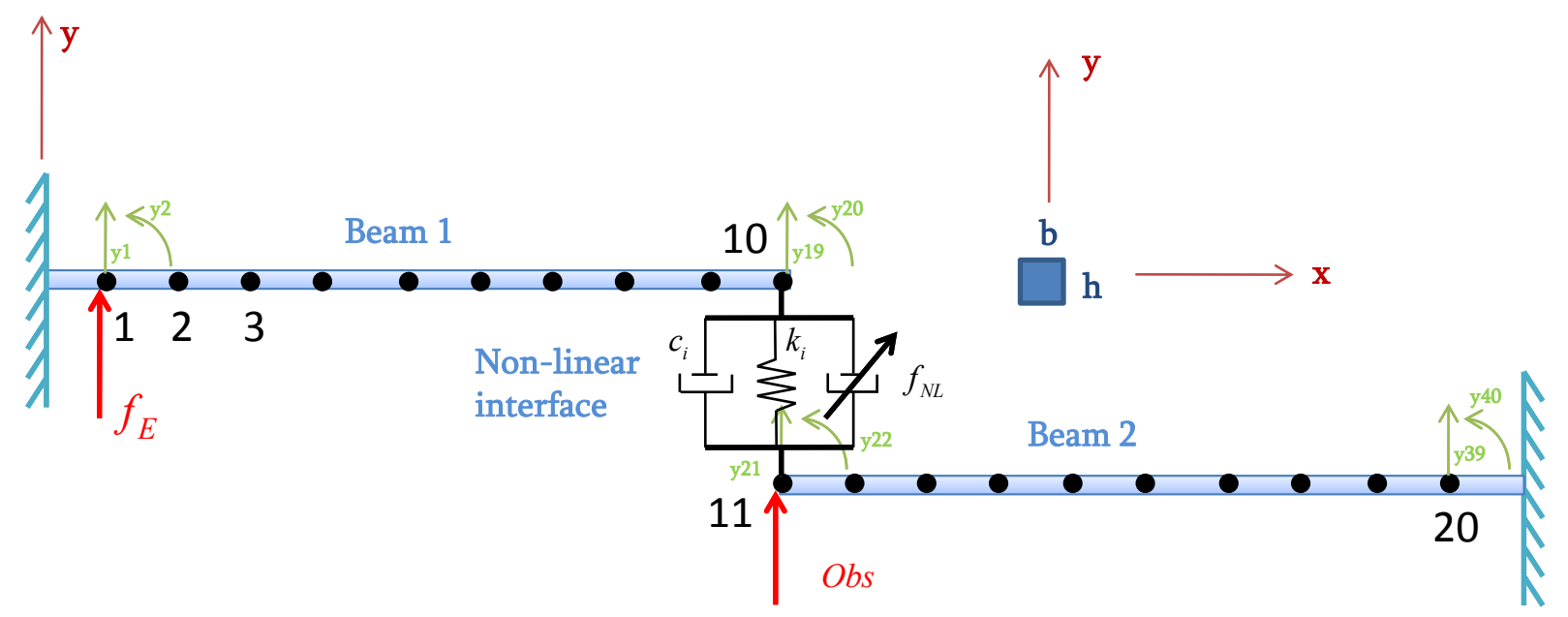

Figure 5: Double-beam structure

The details of the simulation data are summarized in Table 3 . The joint is represented by a nonlinear lumped model composed of spring element $k_{i}$ and a viscous damper element $c_{i}$ and a Van der Pol model governed by the parametric scalar $\alpha$.

Table 3: Physical and geometric properties of the structure and interface parameters

\begin{tabular}{lll||lll}
\hline$E$ & Young's modulus $(\mathrm{GPa})$ & 210 & $k_{i}$ & Linear stiffness $(\mathrm{N} / \mathrm{m})$ & $1 e 4$ \\
$\rho_{s}$ & Mass density $\left(\mathrm{kg} / \mathrm{m}^{3}\right)$ & 7800 & $c_{i}$ & Viscous damper $(\mathrm{N} / \mathrm{m} / \mathrm{s}))$ & 20 \\
$h$ & Beam thikness $(\mathrm{m})$ & 0.02 & $L_{1}$ & Length of the first beam $(\mathrm{m})$ & 0.701 \\
$b$ & Beam width $(\mathrm{m})$ & 0.05 & $L_{2}$ & Length of the second beam $(\mathrm{m})$ & 0.700 \\
$v$ & Poisson ratio & 0.3 & $\alpha$ & scalar parameter of the nonlinear damping $\left(\mathrm{Ns} / \mathrm{m}^{3}\right)$ & $\alpha \in[0-1 e 12]$ \\
\hline
\end{tabular}

The dissipation force is expressed by the following equation:

$$
f_{D}(t, \dot{u})=f_{L N}+f_{N L}=c_{i} \times \dot{y}_{r e l}+\alpha y_{r e l}{ }^{2} \dot{y}_{r e l}
$$

where $y_{\text {rel }}$ is the relative displacements, $\dot{y}_{r e l}$ is the relative velocity at the interface. Figure 6 shows the first four mode shapes for the undamped system of two beams with one interface. From this Figure, we can see that the second mode will have a larger modal damping because it dissipates the most energy, compared to the first one, due to the large relative displacements at the interface. For the same raison the fourth modal damping is higher than the third one.

The modal damping results and eigenfrequencies calculated by the state space method are presented in Table 4 . The influence of the neighborhood of the modes and the extra-diagonal terms of the generalized damping matrix on the dynamic responses of the damped system will then be studied using the proposed perturbation method for both cases of nonlinear and linear localized dissipation. 

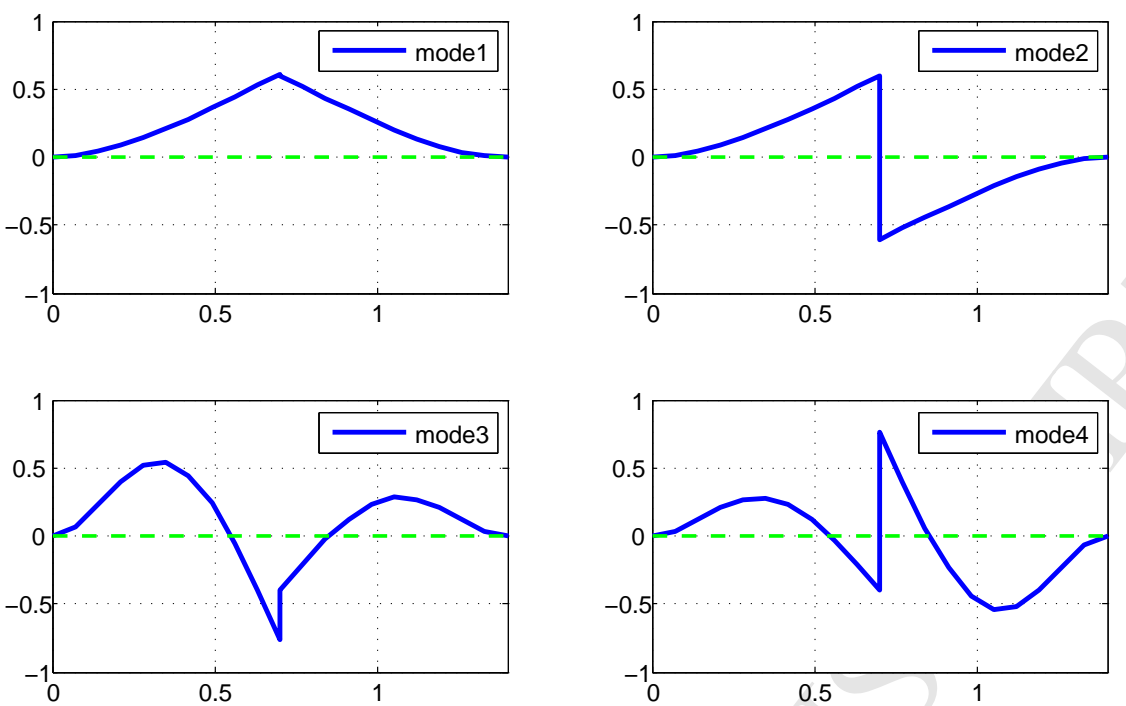

Figure 6: Mode shapes of the double-beam with interface

Table 4: Frequencies and modal damping for the first six modes

\begin{tabular}{lllllll}
\hline Frequency $(\mathrm{Hz})$ & 34.16 & 39.17 & $\mathbf{2 1 4 . 0 1}$ & $\mathbf{2 1 5 . 0 7}$ & 598.91 & 600.65 \\
Modal damping $\xi_{v}(\%)$ & 0.0005 & $\mathbf{5 . 8 3 3 9}$ & 0.0166 & 1.0819 & 0.0599 & 0.3302 \\
\hline
\end{tabular}

\subsubsection{Case 1 : Linear localized damping \\ Impact of modal coupling}

The aim of this section is to investigate the impact of modal coupling. According to the literature [11], this modal coupling is due to two effects : the neighborhood of the modes and the extra-diagonal terms in the generalized damping matrix. The impact of the neglect of these terms can be quantified and corrected using the proposed method.

Figure 7 shows the comparison of the FRFs between the unperturbed and perturbed systems with a perturbation coefficient of $\varepsilon=0.01$, respectively for the first four modes. The estimation of the error in the responses shows that the contribution of extra-diagonal terms is significant when comparing amplitudes between the Reference FRF and the FRF based on the proportional assumption (order 0 ).

The results in the figure 7 respect the criterion of mode separation $R_{j k}=2 \xi_{j} \omega_{j} /\left|\omega_{j}-\omega_{k}\right|<<1$ for decoupled modes, [39, 40]. In fact, modes 1 and 2 are slightly coupled $\left(R_{12}=0.10\right.$ and $\left.R_{21}=0.41\right)$ and display a low error $(0.85 \%$ and $0.68 \%)$, while the strongly coupled modes 3 and $4\left(R_{43}=4\right)$ display a high error $(87.5 \%)$. These results illustrate that the proportional damping assumption can yield results with acceptable errors, as it is the case for the first two modes. However, neglecting the contribution of the off-diagonal terms can lead to significant amplitude errors, as is the case for modes 3 and 4.

The generalized damping matrix in Equation (41) expresses the importance of extra-diagonal terms and their effects on the accuracy of the response. Nevertheless, the response of the first mode is not affected by these terms, as the modal damping is quasi-null. The second mode is insensitive to extra-diagonal terms since it has a high modal damping (5.83\%) compared to other modes. The combined effects of the modal neighborhood and the extra-diagonal terms of the matrix lead to significant errors on the responses of modes 3 and 4 . The effects of extra-diagonal terms will be highlighted again in the nonlinear simulation (Subsection 4.2.2).

$$
\beta=\phi^{T} C \phi=\left(\begin{array}{cccc}
0.0024 & 0.2605 & -0.0797 & -0.2525 \\
0.2605 & 28.7087 & -8.7818 & -27.8346 \\
& & 2.6863 & 8.5144 \\
\text { Sym } & & & 26.9871
\end{array}\right)
$$




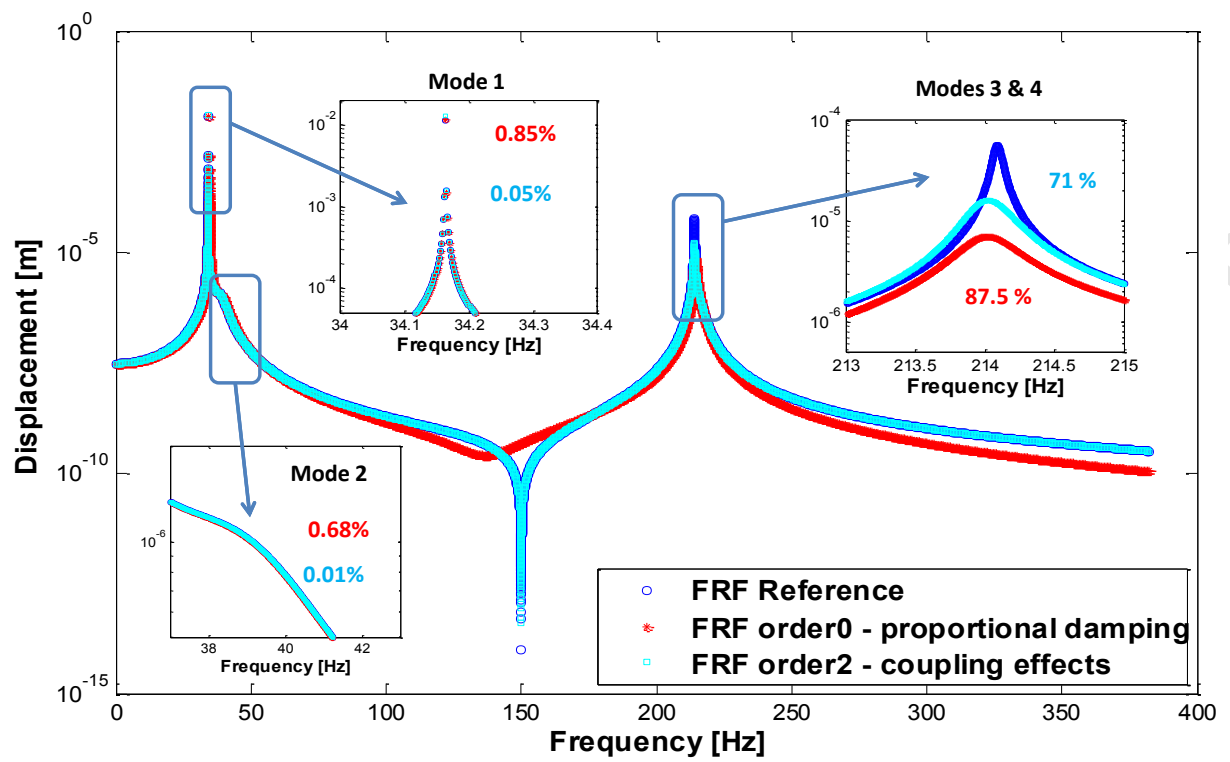

Figure 7: Comparison of FRFs at interface dof 21 for $\varepsilon=0.01$. (a) Mode $1,\left(\varepsilon_{y}^{\text {Order } 0}=0.68 \%\right)$. (b) Mode $2,\left(\varepsilon_{y}^{\text {Order } 0}=0.85 \%\right)$. (c) Modes $3 \&$ $4,\left(\varepsilon_{y}^{\text {Order } 0}=87.5 \%\right)$. Reference method: FRF based on an inversion of full matrix using Equation (35). Order0 : FRF based on the proportional assumption given by Equation (33). Order2 : FRF based on the perturbed method taking into account the coupled terms using Equation (34).

In order to imporouve the accuracy of the results for modes 3 and 4, the perturbation method is applied with high order terms (Figure 8). Even if this case presents extreme coupling conditions $(\xi>5.8 \%$ for the second mode and $R_{43}=4$ ), the proposed method reduces the error resulting from the proportional damping assumption, from $87.5 \%$ to $2.1 \%$ by increasing the perturbation order up to 30 . It is important to note that increasing the perturbation order improves the accuracy without increasing the computational cost as explained previously in section 3.2, when using Equation (34).

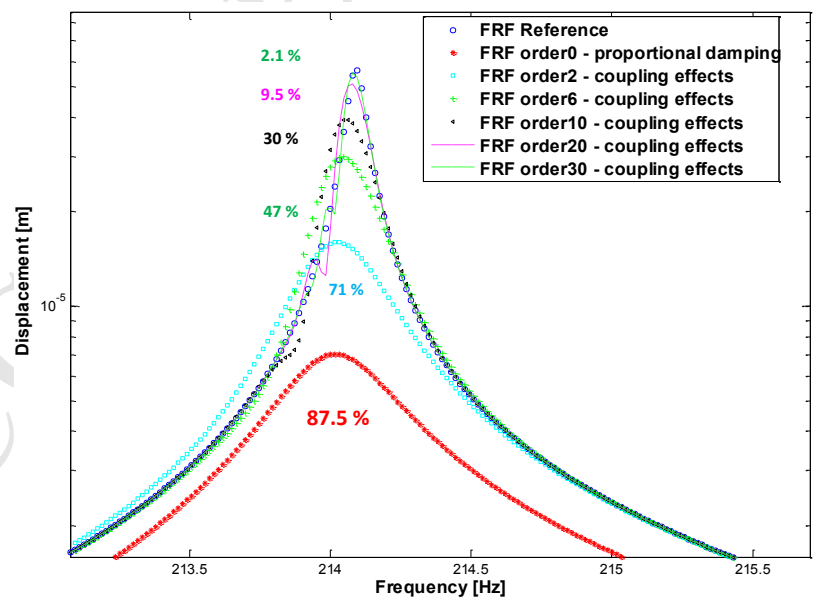

Figure 8: Zoom in the neighbourhood of modes 3,4. Reference method: FRF based on an inversion of full matrix using Equation (35). Order0 : FRF based on the proportional assumption given by Equation (33). Order $n$ : FRF based on the perturbed method taking into account the coupled terms using Equation (34). 


\section{Impact of the localized damping level}

Consider again the two-beam system connected by a localized dissipative interface as shown in Figure 5. In this section, the effect of the localized damping level on the damping prediction will be examined. Five numerical simulations are performed, for each order, with increasing levels of viscous damping $\left(c_{i}=2,5,10,15\right.$ and $\left.20 \mathrm{Ns} / \mathrm{m}\right)$ leading to increasing dissipated energies.

Now if we just focus on the third and fourth modes for example, we can investigate the influence of the localized damping level. Table 5 shows the evolution of the relative errors with the proportional assumption (order 0 ) and the correction of these errors using the proposed method (order $2 \rightarrow 30$ ). It can be seen that the error increases with the increase of the value of the localized dissipation index $I_{L N}$ for all orders.

Particularly, the order 2 is able to correct the errors due to the proportional damping hypothesis. For instance, in the case $I_{L N}=10$, the error is reduced from $29.50 \%$ down to $2.31 \%$. Neverthless, if the ratio $I_{L N}$ increases (the localized dissipation increases), the order must be increased in order to reach a sufficient accuracy. In the case $I_{L N}=40$, at the $30^{\text {th }}$ order, the error is drastically reduced from $87.5 \%$ down to $2.1 \%$.

Table 5: Amplitude Error (\%) for modes 3 and 4

\begin{tabular}{cc||c||cc||c||}
\hline Coef $c_{i}$ & 2 & 5 & 10 & 15 & 20 \\
Ratio $I_{L N}$ & 5 & 10 & 20 & 30 & 40 \\
\hline$(0)$ & $\mathbf{1 0 . 5 0}$ & $\mathbf{2 9 . 5 0}$ & $\mathbf{6 2 . 9 7}$ & $\mathbf{7 9 . 8 3}$ & $\mathbf{8 7 . 5}$ \\
$(2)$ & $\mathbf{2}$ & 2.31 & 28.70 & 56.89 & 71 \\
$(6)$ & 0.11 & $\mathbf{1 . 3 9}$ & 2.71 & 24 & 47 \\
$(10)$ & 0.01 & 0.47 & $\mathbf{0 . 9 1}$ & 12.28 & 30 \\
$(20)$ & 0.001 & 0.001 & 0.84 & $\mathbf{2 . 4}$ & 9.5 \\
$(30)$ & $\mathbf{0}$ & $\mathbf{0}$ & $\mathbf{0}$ & $\mathbf{0}$ & $\mathbf{2 . 1}$ \\
\hline
\end{tabular}

The main advantage of the proposed method in the case of linear behaviour is its capacity to correct the error induced by neglecting the coupled terms with a reduced computational cost.

\section{Convergence remarks}

The reference solution is not always known in the real applications. But even in this case a residual error for the approximate solution can be evaluated a posteriori without calculating the reference solution. By using the convergence indicator $\varepsilon_{f}$, the minimum order that guarantee an acceptable residual error can be calculated. For instance, when $I_{L N}=5$, if a limit error of residual force equal to $\varepsilon_{\text {limit }}=0.1 \%$, the second order response of the perturbed method gives a convergence indicator equal to $\varepsilon_{f}=0.01 \%<\varepsilon_{\text {limit }}$. So, the second order is sufficient, in this case, to predict the reference response with an acceptable level error $\varepsilon_{y} \leq 2 \%$.

\subsubsection{Case 2 : Nonlinear localized damping}

To highlight the coupling from nonlinear localized damping, the first six eigenfrequencies were separated by modifying the length of the second beam $L 2=0.63 \mathrm{~m}$ instead of $L 2=0.70 \mathrm{~m}$. The modal damping and eigenfrequencies are presented in Table 6.

Table 6: Frequencies and modal damping for the first six modes

\begin{tabular}{lllllll}
\hline Frequency $(\mathrm{Hz})$ & 35.96 & 45.19 & 214.23 & 265.10 & 598.92 & 741.57 \\
Modal damping $\xi_{v}(\%)$ & 1.43 & 4.21 & 0.54 & 0.50 & 0.19 & 0.17 \\
\hline
\end{tabular}

From Figure 9, it can be seen that, the order $(0,0)$ which corresponds to the proportional damping hypothesis has a $14.23 \%$ response level error. This error decreases if we consider the linear coupling. The order (0.1) presents an error equals to $6.75 \%$. Taking into account the presence of uncoupled nonlinearity, the response is clearly corrected and the error of the order (1.0) is reduced to $3.14 \%$. Finally, if the coupled nonlinearity is considered, the order $(1,1)$ presents in this case $2.98 \%$ of error with respect to the nonlinear reference. 


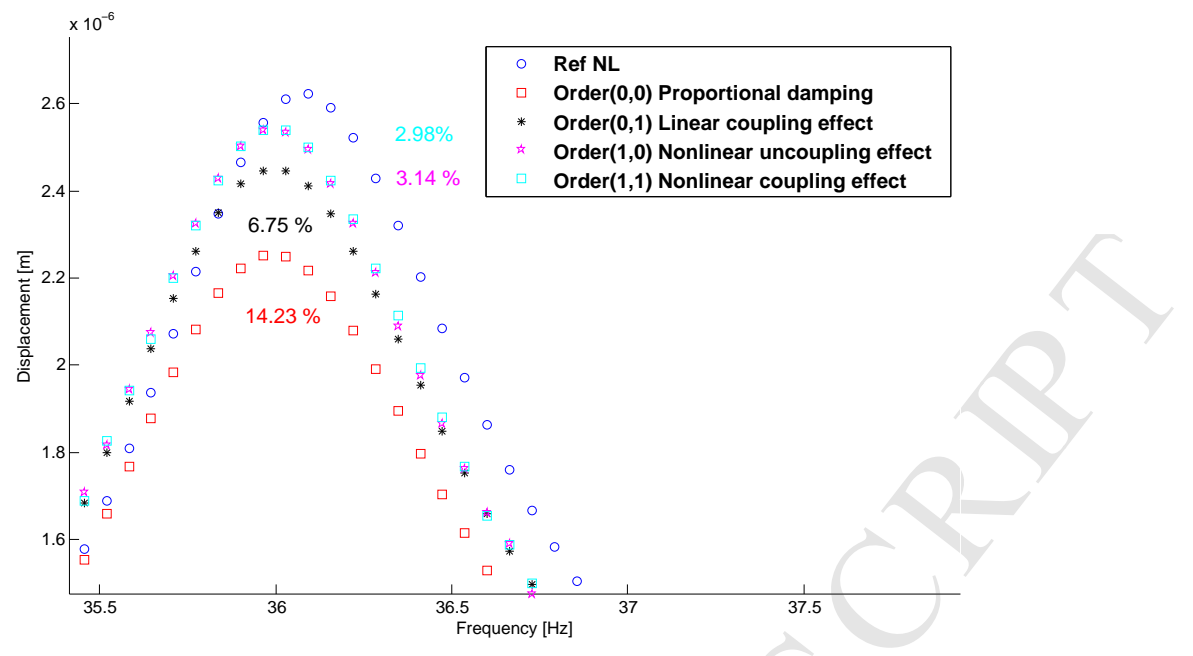

Figure 9: Frequency response of the nonlinear model, nonlinear coupling index $I_{N L}=5 \%$, focus on the first mode: $\circ$ Reference, $\square$ Proportional damping, * Order $(0,1)$ - Linear coupling effect, $\star$ Order $(1,0)$ - nonlinear uncoupling effect, $\square$ Order $(1,1)$ - nonlinear coupling effect

The Table 7 shows that the error increases with the increase of the value of the nonlinear dissipation index $I_{N L}$ for all the orders. For the case of few nonlinearity, when $I_{N L}=5 \%$, the error drops from $14.23 \%$ to $2.98 \%$, and from $21 \%$ to $3.5 \%$ in the case of $I_{N L}=20 \%$. The order does not completely compensate the dynamic response error when the NL ratio is above $20 \%$. But the proposed method is of high interest in weakly nonlinear structures. In this case the order $(1,1)$ is sufficient, when $I_{N L} \leq 20 \%$, in order to get an accurate result of dynamic response (less than $4 \%$ of level error) using the perturbation method. Finally, the proposed method is able to predict the maximal level error due to the neglect of coupling linear and nonlinear terms. Also, the perturbation method is able to reduce these errors and generate an accurate result in the case of non-proportional nonlinear dissipation.

Table 7: Amplitude Error (\%)

\begin{tabular}{c||c||ccc}
\hline Ratio $I_{N L}$ & 5 & 10 & 20 & 30 \\
\hline$(0,0)$ & $\mathbf{1 4 . 2 3}$ & $\mathbf{1 5 . 6 8}$ & $\mathbf{2 0 . 9 8}$ & $\mathbf{2 5 . 4 5}$ \\
$(0,1)$ & 6.75 & 8.46 & 14.11 & 19.06 \\
$(1,0)$ & 3.14 & 3.70 & 5.79 & 10.02 \\
$(1,1)$ & $\mathbf{2 . 9 8}$ & $\mathbf{3 . 3 3}$ & $\mathbf{3 . 5}$ & $\mathbf{9 . 2 0}$ \\
\hline
\end{tabular}

\section{Discussion and conclusions}

The limitation of the modal damping assumption was firstly investigated when dealing with both linear and nonlinear localized dissipation. Then an estimation and correction method was proposed and illustrated with two numerical examples in order to investigate the importance of the coupling terms on the frequency response of the structure. Through an extended perturbation theory, we show that the coupling part of the restoring force can affect the frequency response level near the resonances.

The proposed method based on a double perturbation formulation is able to correct both amplitude and phase shift when switching to higher orders. For instance, in the linear case of the double-beam structure, the proposed method until order 6 allows to reduce the error for strongly coupled modes 3 and 4, from $29.5 \%$ down to $1.39 \%$ in the case of low localized dissipation $\left(I_{L N}=10 \%\right)$. Even for high level of localized dissipation $\left(I_{L N}=40 \%\right)$, due to the proposed method until order 30 , the error was drastically reduced from $87.5 \%$ down to $2.1 \%$ for these modes. In the nonlinear case, the order $(1,1)$ is sufficient and can generate responses with good accuracy (less than $4 \%$ of level error) for high nonlinearity $\left(I_{N L}=30 \%\right)$. 
In the specific case of linear localized dissipation, the proposed method is able to quantify and correct the error due to the modal coupling with a low computational cost. In the general case of nonlinear localized dissipation, the proposed method is accurate but the computational cost remains reasonable only for small size FEM.

Finally, to apply the perturbation method for industrial large size FEM, it is advisable to work with metamodels which can be used locally, so that the nonlinear resolution taking into account the coupling becomes possible with a reasonable computational cost. Future work will include the development of adapted metamodels which are compatible with our proposed methodology in order to estimate the damping of nonlinear large size structures.

\section{Acknowledgments}

The authors are grateful to the Agence Nationale de La Recherche (ANR) for their financial support of this project (contract ANR-12-MONU-00016-01). The Authors declare that there is no conflict of interest. 


\section{References}

[1] Caignot, A., Ladeveze, P., Neron, D., \& Durand, J. F. (2010). Virtual testing for the prediction of damping in joints. Engineering Computations, 27(5), 621-644.

[2] Rayleigh, J. W. S. B. (1896). The theory of sound (Vol. 2). Macmillan.

[3] Hammami, C., Balmes, E., \& Guskov, M. (2016). Numerical design and test on an assembled structure of a bolted joint with viscoelastic damping. Mechanical Systems and Signal Processing, 70, 714-724.

[4] Adhikari, S. (2013). Structural Dynamic Analysis with Generalized Damping Models: Analysis. John Wiley \& Sons.

[5] Bograd, S., Reuss, P., Schmidt, A., Gaul, L., \& Mayer, M. (2011). Modeling the dynamics of mechanical joints. Mechanical Systems and Signal Processing, 25(8), 2801-2826.

[6] Ahmadian, H., \& Jalali, H. (2007). Identification of bolted lap joints parameters in assembled structures. Mechanical Systems and Signal Processing, 21(2), 1041-1050.

[7] Mead, D. J. (1999). Passive vibration control. John Wiley \& Sons Inc.

[8] Goodman, L. E., \& Klumpp, J. H. (1956). Analysis of slip damping with reference to turbine-blade vibration. Journal of Applied Mechanics, 23, 421-429.

[9] Beards, C. F., \& Williams, J. L. (1977). The damping of structural vibration by rotational slip in joints. Journal of Sound and Vibration, 53(3), 333-340.

[10] Ungar, E. E. (1973). The status of engineering knowledge concerning the damping of built-up structures. Journal of Sound and Vibration, 26(1), 141-154.

[11] Sinou, J. J., \& Jezequel, L. (2007). Mode coupling instability in friction-induced vibrations and its dependency on system parameters including damping. European Journal of Mechanics-A/Solids, 26(1), 106-122.

[12] Peyret, N., Dion, J. L., Chevallier, G., \& Argoul, P. (2010). Micro-slip induced damping in planar contact under constant and uniform normal stress. International Journal of Applied Mechanics, 2(02), 281-304.

[13] Festjens, H., Chevallier, G., \& Dion, J. L. (2013). A numerical tool for the design of assembled structures under dynamic loads. International Journal of Mechanical Sciences, 75, 170-177.

[14] . Chen, W., \& Deng, X. (2005). Structural damping caused by micro-slip along frictional interfaces. International Journal of Mechanical Sciences, 47(8), 1191-1211.

[15] Festjens, H., Chevallier, G., \& Dion, J. L. (2014). Nonlinear model order reduction of jointed structures for dynamic analysis. Journal of Sound and Vibration, 333(7), 2100-2113.

[16] Dahl, P. R. (1968). A solid friction model. The Aerospace Corporation, EI Segundo (pp. 3107-3118). CA, Tech. Rep. TOR-158.

[17] Iwan, W. D. (1966). A distributed-element model for hysteresis and its steady-state dynamic response. Journal of Applied Mechanics, 33(4), 893-900.

[18] Segalman, D. J. (2005). A four-parameter Iwan model for lap-type joints. Journal of Applied Mechanics, 72(5), $752-760$.

[19] Jalali, H. (2016). Linear contact interface parameter identification using dynamic characteristic equation. Mechanical Systems and Signal Processing, 66, 111-119.

[20] Buks, E., \& Roukes, M. L. (2002). Electrically tunable collective response in a coupled micromechanical array. Journal of Microelectromechanical Systems, 11(6), 802-807.

[21] Kacem, N., Hentz, S., Pinto, D., Reig, B., \& Nguyen, V. (2009). Nonlinear dynamics of nanomechanical beam resonators: improving the performance of NEMS-based sensors. Nanotechnology, 20(27), 275501.

[22] Gutschmidt, S., \& Gottlieb, O. (2012). Nonlinear dynamic behavior of a microbeam array subject to parametric actuation at low, medium and large DC-voltages. Nonlinear Dynamics, 67(1), 1-36.

[23] Lifshitz, R., \& Cross, M. C. (2003). Response of parametrically driven nonlinear coupled oscillators with application to micromechanical and nanomechanical resonator arrays. Physical Review B, 67(13), 134302.

[24] Bogoliubov, N. N., \& Mitropolski, Y. A. (1961). Asymptotic methods in the theory of nonlinear oscillations. Asymptotic Methods in the Theory of NonLinear Oscillations, by NN Bogoliubov and YA Mitropolski. New York: Gordon and Breach, $1961 ., 1$.

[25] Nayfeh, A. H. (2011). Introduction to perturbation techniques. John Wiley \& Sons.

[26] Chaigne, A., \& Kergomard, J. (2008). Acoustique des instruments de musique, Belin.

[27] Caughey, T. K., \& Okelly, M. E. J. (1965). Classical normal modes in damped linear dynamic systems. Journal of Applied Mechanics, 32(3), $583-588$.

[28] Adhiakri, S. (2001). Classical normal modes in nonviscously damped linear systems. AIAA journal, 39(5), 978-980.

[29] Ungar, E. E., \& Kerwin Jr, E. M. (1962). Loss factors of viscoelastic systems in terms of energy concepts. The Journal of the Acoustical Society of America, 34(7), 954-957.

[30] Johnson, C. D., \& Kienholz, D. A. (1982). Finite element prediction of damping in structures with constrained viscoelastic layers. AIAA journal, 20(9), 1284-1290.

[31] Hu, B. G., Dokainish, M. A., \& Mansour, W. M. (1995). A modified MSE method for viscoelastic systems: a weighted stiffness matrix approach. Journal of vibration and acoustics, 117(2), 226-231.

[32] Krifa, M., Bouhaddi, N., \& Cogan, S. (2015). Estimation of modal damping for structures with localized dissipation. In Special Topics in Structural Dynamics, Volume 6 (pp. 179-191). Springer International Publishing.

[33] Bandstra, J. P. (1983). Comparison of equivalent viscous damping and nonlinear damping in discrete and continuous vibrating systems. Journal of Vibration, Acoustics, Stress, and Reliability in Design, 105(3), 382-392.

[34] Petyt, M. (2010). Introduction to finite element vibration analysis. Cambridge university press.

[35] Liu, K., Kujath, M. R., \& Zheng, W. (2000). Quantification of non-proportionality of damping in discrete vibratory systems. Computers \& Structures, 77(5), 557-570.

[36] Bhaskar, A. (1995). Estimates of errors in the frequency response of non-classically damped systems. Journal of Sound and Vibration, 184(1), $59-72$. 
[37] Tong, M., Liang, Z., \& Lee, G. C. (1994). An index of damping non-proportionality for discrete vibrating systems. Journal of Sound and Vibration, 174(1), 37-55.

[38] Gradin, M., \& Rixen, D. J. (2014). Mechanical vibrations: theory and application to structural dynamics. John Wiley \& Sons.

[39] Hasselman, T. K. (1976). Modal coupling in lightly damped structures. AIAA Journal, 14(11), 1627-1628.

[40] Balmes, E. (1997). New results on the identification of normal modes from experimental complex modes. Mechanical Systems and Signal Processing, 11(2), 229-243 